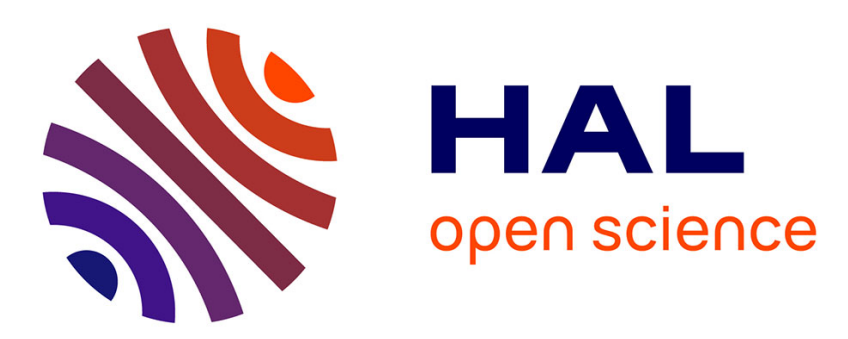

\title{
A thermo-mechanical modelling of the Tribological Transformations of Surface
}

\author{
Grégory Antoni, Thierry Désoyer, Frédéric Lebon
}

\section{To cite this version:}

Grégory Antoni, Thierry Désoyer, Frédéric Lebon. A thermo-mechanical modelling of the Tribological Transformations of Surface. Comptes Rendus Mécanique, 2009, 337 (9-10), pp.653-658. 10.1016/j.crme.2009.09.003 . hal-00418398

\section{HAL Id: hal-00418398 \\ https://hal.science/hal-00418398}

Submitted on 30 Jun 2010

HAL is a multi-disciplinary open access archive for the deposit and dissemination of scientific research documents, whether they are published or not. The documents may come from teaching and research institutions in France or abroad, or from public or private research centers.
L'archive ouverte pluridisciplinaire HAL, est destinée au dépôt et à la diffusion de documents scientifiques de niveau recherche, publiés ou non, émanant des établissements d'enseignement et de recherche français ou étrangers, des laboratoires publics ou privés. 
This paper has been published in Comptes Rendus Mécanique, Vol. 337, pp. 653658, 2009 (DOI : 10.1016/j.crme.2009.09.003).

\section{A thermo-mechanical modelling of the Tribological Transformations of Surface.}

Grégory ANTONI (antoni@lma.cnrs-mrs.fr), Université de Provence \& LMA (UPR 7051-CNRS), 31 chemin Joseph Aiguier, 13402 Marseille cedex 20, France.

Thierry DÉSOYER (thierry.desoyer@ec-marseille.fr), EC-Marseille \& LMA (UPR 7051-CNRS), Technopôle de Château-Gombert, 38 rue Joliot Curie, 13451 Marseille Cedex 20, France.

Frédéric LEBON (lebon@lma.cnrs-mrs.fr), Université de Provence \& LMA (UPR 7051-CNRS), 31 chemin Joseph Aiguier, 13402 Marseille cedex 20, France.

Abstract The Tribological Transformations of Surface (TTS) are observed on samples of certain steels undergoing repeated compressive loadings. They correspond to a permanent, solid-solid phase transformation localized on the surfaces of the sample on which the loading is applied. The main hypothesis of the study is that TTS are not only due to the mechanical loading but also to the thermal loading which is associated to. Thus, a thermo-mechanical model is first proposed in the present paper, which is inspired by previous works on TRansformation Induced Plasticity (TRIP). The potentialities of the model are also briefly illustrated by a simple $1 D$ example.

Keywords: Tribological Transformations of Surface (TTS); Transformation Induced Plasticity (TRIP); Thermo-mechanical Modelling; Cyclic Compression.

Résumé Les Transformations Tribologiques de Surface (TTS) sont observées sur des échantillons de certains aciers soumis à des chargements compressifs répétés. Elles correspondent à des changements de phase solide-solide irréversibles localisés sur les surfaces mécaniquement sollicitées des échantillons. L'hypothèse centrale de cette étude est que les TTS ne sont pas dus au seul chargement mécanique mais également au chargement thermique qui l'accompagne. Un modèle thermo-mécanique est ainsi proposé, qui s'inspire de travaux antérieurs sur la plasticité de transformation (TRIP). Un exemple ( $1 D)$ illustratif des potentialités de ce modèle est également présenté.

Mots-clés : Transformations Tribologiques de Surface (TTS) ; Plasticité de Transformation (TRIP) ; Modélisation Thermomécanique ; Compression Cyclique.

\section{Introduction}

The Tribological Transformations of Surface (TTS) are permanent, quasi-surfacic solid-solid phase transformations, e.g. ferrito-pearlitic-martensitic transformations. They are observed only on certain steels and, in most of the cases, for repeated compressive loadings. They are characterized by a thin, "white" layer progressively appearing, in proportion to the number of loading cycles, on the surfaces - and in their immediate vicinity - of the sample where the loading is applied (see e.g. Baumann et al., [1]; Eleöd et al., [2]). In the worst cases, and for a number of cycles depending on both the material and the loading, TTS can eventually lead to the emergence of a crack, due to strong strain incompatibilities between the quasi-surfacic, "white" phase (e.g. martensite) and the volumetric, "bulk" phase (e.g. ferrito-pearlite).

Whatever the loading apparatus is, more or less important friction effects are systematically associated to compressive loadings. Hence, more or less important temperature variations are induced by such loadings on the surfaces of the sample where the loading is applied, i.e. where TTS appear. These temperature variations, however, are clearly too small for generating alone solid-solid phase transformations. On the other hand, they induce thermal stresses which, as small as they are, make the actual state of stress greater than this due to the only mechanical loading, especially on the surfaces - and in their immediate vicinity - where the loading is applied. It seems then not unrealistic to assume that TTS result from a thermo-mechanical coupling: this is actually the main assumption underlying the present study. It can be underlined that this is in line with a comment made by Baumann et al. in [1] that "... there is some [experimental] evidence to suggest that the [Tribological] Transfomation [of Surface] may be produced by normal and shear stresses superimposed with high thermal stresses limited to a small surface layer."

The paper is organized as follows: a thermodynamically consistent, thermo-mechanical model is presented in Section 2. It is based on previous works (see e.g. Taleb and Sidoroff, [3]) on TRansformation 
Induced Plasticity (TRIP), which, however, are here extended for a thermo-mechanical coupling, relevant for TTS, to be taken into account. The potentialities of the model are illustrated in Section 3 on the simple $1 D$ example of a bar submitted to a cyclic thermo-mechanical loading.

\section{Solid-solid phase transformation and associated thermo- mechanical coupling: a phenomenological modelling}

Surfacic or not, the permanent solid-solid phase transformations are generally associated to so-called "anomalous" plastic strains, in the sense that these irreversible strains appear for states of stress such that the associated Von Mises or Tresca stress is much lower than the initial yield strength of the softest phase. This phenomenon is known as TRansformation Induced Plasticity (TRIP). It has been widely studied and, from a purely mechanical point of view, sucessfully modelled, see e.g. Taleb and Sidoroff, [3]. The phenomenological model detailed in this Section is clearly based on these previous works. These are simply extended for the main assumption of the study- TTS result from a thermo-mechanical coupling, see also Section 1 - to be well taken into account. Thus, the absolute temperature $T$ is explicitely considered as a state variable. In the same way as in [3], the following state variables are also considered: i - the infinitesimal strain tensor $\epsilon$; ii - the transformation induced, plastic strain tensor $\epsilon^{p z}$; iii - the volume fraction of the "new" phase (i.e. that formed by the transformation), $z \in[0,1]$. Whenever the transformation is not initiated, we then have $z=0$ and $\epsilon^{p z}=0$. Later on, the solid phase corresponding to $z=0$ will be called phase 0 and that correponding to $z=1$ (i.e. to the "new" phase), phase 1 .

Denoting by $T_{i}$ the initial temperature and assuming, for the sake of simplicity, that the considered material is initially non-transformed (i.e. $z_{i}=0$ and $\epsilon_{i}^{p z}=0$ ), the following simple expression can then be considered for the local state potential of (per unit mass) free energy:

$$
\begin{gathered}
\psi\left(T, \boldsymbol{\epsilon}, \boldsymbol{\epsilon}^{p z}, z\right)=-\frac{C_{\epsilon}}{2 T_{i}}\left(T-T_{i}\right)^{2}-\frac{(3 \lambda+2 \mu)}{\rho_{i}}\left(\alpha_{0}(1-z)+\alpha_{1} z\right)\left(T-T_{i}\right) \operatorname{Tr}\left(\boldsymbol{\epsilon}-\boldsymbol{\epsilon}^{p z}\right) \\
+\frac{\lambda}{2 \rho_{i}}\left(\operatorname{Tr}\left(\boldsymbol{\epsilon}-\boldsymbol{\epsilon}^{p z}\right)\right)^{2}+\frac{\mu}{\rho_{i}}\left(\left(\boldsymbol{\epsilon}-\boldsymbol{\epsilon}^{p z}\right):\left(\boldsymbol{\epsilon}-\boldsymbol{\epsilon}^{p z}\right)\right)+\frac{\beta}{2 \rho_{i}} z^{2}+\psi_{i}
\end{gathered}
$$

where $C_{\epsilon}>0$ is the specific heat capacity (the same for the two phases), $\mu>0$ and $\lambda>-(2 / 3) \mu$, the Lamé constants (the same for the two phases), $\alpha_{0}>0$ (resp. $\alpha_{1}>0$ ), the coefficient of thermal expansion of phase 0 (resp. of phase 1), and where $\beta>0$ is a material-parameter characterizing the energy stored by the material during the phase transformation: from this point of view, $z$ can then be also considered as a hardening variable. In Eq. (1), $\rho_{i}$ (resp. $\psi_{i}$ ) is the initial density (resp. the initial, per unit mass, free energy) of the material. Note that, in a very first approximation, the volume change due to phase transformation is not taken into account in Eq. (1).

Taking $e$ to denote the (per unit mass) internal energy, and noting that $e=\psi+T s$, where $s$ is the (per unit mass) entropy, the local expression of the first principle of the Thermodynamics simply reads (see e.g. Garrigues, [4]):

$$
\rho \dot{e}=\boldsymbol{\sigma}: \dot{\boldsymbol{\epsilon}}-\operatorname{div}(\boldsymbol{q}) \Leftrightarrow \rho T \dot{s}+\operatorname{div}(\boldsymbol{q})=\boldsymbol{\sigma}: \dot{\boldsymbol{\epsilon}}-\rho s \dot{T}-\rho \dot{\psi}
$$

where $\boldsymbol{q}$ is the heat flux vector (for the sake of simplicity, the rate of remote heat supply is omitted) and $\boldsymbol{\sigma}$, the Cauchy stress tensor. The second principle of Thermodynamics must be satisfied (see e.g. [4]): $\mathrm{i}$ - whatever the local, thermomecanical state of the material may be, $\left(T, \boldsymbol{\epsilon}, \boldsymbol{\epsilon}^{p z}, z\right)$; ii - whatever the local evolution undergone by the material, $(\dot{T}, \dot{\epsilon})$; iii - whatever the temperature gradient locally acting on the material, $\nabla T$, i.e.:

$$
-\frac{1}{T} \boldsymbol{q} \cdot \boldsymbol{\nabla} T+\rho T \dot{s}+\operatorname{div}(\boldsymbol{q}) \geq 0 \quad \forall\left(T, \boldsymbol{\epsilon}, \boldsymbol{\epsilon}^{p z}, z\right), \forall(\dot{T}, \dot{\boldsymbol{\epsilon}}), \forall \boldsymbol{\nabla} T
$$

Upon combining Eqs. (1), (2) and (3), we immediatly obtain the Clausius-Duhem inequality:

$-\frac{1}{T} \boldsymbol{q} \cdot \boldsymbol{\nabla} T-\rho\left(s+\frac{\partial \psi}{\partial T}\right) \dot{T}+\left(\boldsymbol{\sigma}-\rho \frac{\partial \psi}{\partial \boldsymbol{\epsilon}}\right): \dot{\boldsymbol{\epsilon}}-\rho \frac{\partial \psi}{\partial \boldsymbol{\epsilon}^{p z}}: \dot{\boldsymbol{\epsilon}}^{p z}-\rho \frac{\partial \psi}{\partial z} \dot{z} \geq 0 \quad \forall\left(T, \boldsymbol{\epsilon}, \boldsymbol{\epsilon}^{p z}, z\right), \forall(\dot{T}, \dot{\boldsymbol{\epsilon}}), \forall \nabla T$

Assuming that neither $\dot{\boldsymbol{\epsilon}}^{p z}$, nor $\dot{z}$ depend on $\dot{T}$, and due to the fact that the entropy, $s$, is a state function, i.e. depends only on the state variables, a necessary and sufficient condition for inequality Eq. (4) to be systematically satisfied is: $s=-\partial \psi / \partial T$. Two other classical, sufficient conditions for Eq. (4) to be systematically satisfied will also be considered in the present study, namely:

$$
\boldsymbol{q}=-k \boldsymbol{\nabla} T \quad ; \quad \boldsymbol{\sigma}=\rho \frac{\partial \psi}{\partial \boldsymbol{\epsilon}}
$$


where $k>0$ is the thermal conductivity (the same for the two phases). Equality Eq. (5)-2 indicates that the viscoelastic effects are not taken into account. From Eq. (5) and the expression of the entropy, and assuming that neither $\dot{\boldsymbol{\epsilon}}^{p z}$, nor $\dot{z}$ depend on $\nabla T$, a sufficient condition for Eq. (4) to be systematically satisfied is then:

$$
-\rho \frac{\partial \psi}{\partial \boldsymbol{\epsilon}^{p z}}: \dot{\boldsymbol{\epsilon}}^{p z}-\rho \frac{\partial \psi}{\partial z} \dot{z} \geq 0 \quad \forall\left(T, \boldsymbol{\epsilon}, \boldsymbol{\epsilon}^{p z}, z\right), \forall \dot{\boldsymbol{\epsilon}}
$$

or, in an equivalent way, according to Eq. (5)-2 and the expression of the free energy, see Eq. (1), from which $\rho\left(\partial \psi / \partial \boldsymbol{\epsilon}^{p z}\right)=-\rho(\partial \psi / \partial \boldsymbol{\epsilon})$ :

$$
\boldsymbol{\sigma}: \dot{\boldsymbol{\epsilon}}^{p z}-\rho \frac{\partial \psi}{\partial z} \dot{z} \geq 0 \quad \forall\left(T, \boldsymbol{\epsilon}, \boldsymbol{\epsilon}^{p z}, z\right), \forall \dot{\boldsymbol{\epsilon}}
$$

The evolution equations of the so-called "internal", state variables $\epsilon^{p z}$ and $z$ are then classically defined by: i - a TRIP yield surface $f(\sigma,-\rho \partial \psi / \partial z)=0$; ii - a viscoplastic multiplier $\Lambda \geq 0$; iii - a normality rule (with respect to the TRIP yield surface), such that:

$$
\begin{gathered}
f\left(\boldsymbol{\sigma},-\rho \frac{\partial \psi}{\partial z}\right)=\sigma_{e q}-\left(\exp \left(\frac{T_{i}-T}{T_{i}}\right) \sigma_{i}^{p}+\gamma^{p} \rho \frac{\partial \psi}{\partial z}\right) \quad \text { with } \quad \sigma_{e q}=\left(\frac{3}{2} \operatorname{dev}(\boldsymbol{\sigma}): \operatorname{dev}(\boldsymbol{\sigma})\right)^{\frac{1}{2}} \\
\dot{\boldsymbol{\epsilon}}^{p z}=\Lambda \frac{\partial f}{\partial \boldsymbol{\sigma}}=\frac{3}{2 \sigma_{e q}} \Lambda \operatorname{dev}(\boldsymbol{\sigma}) \quad ; \dot{z}=\Lambda \frac{\partial f}{\partial\left(-\rho \frac{\partial \psi}{\partial z}\right)}=\gamma^{p} \Lambda \quad \text { with } \quad \Lambda=\frac{1-z}{\chi^{p}}\left\langle f\left(\boldsymbol{\sigma},-\rho \frac{\partial \psi}{\partial z}\right)\right\rangle
\end{gathered}
$$

where $\langle$.$\rangle denotes the MacCauley brackets, i.e. \langle x\rangle=x$ when $x \geq 0$ and $\langle x\rangle=0$ when $x<0$. In Eq. (8), $\sigma_{i}^{p}>0$ is the TRIP yield strength when $T=T_{i}$. It is here assumed that this yield strength slowly decreases as the temperature increases (exponential term in Eq. (8)). The material-parameters $\chi^{p}$ and $\gamma^{p}$ (which acts as a hardening parameter) are also assumed to be strictly positive. Thus, due to the fact that $z \in[0,1]$, the viscoplastic multiplyer $\Lambda$ defined in Eq. (8) is ever non-negative. Furthermore, since $\dot{z}=\gamma^{p} \Lambda, z$ cannot decrease which is in agreement with the fact that the solid-solid phase transformations considered in this study are permanent. Note also that, as soon as the material is fully transformed, i.e. as soon as $z=1$, the viscoplastic multiplyer is zero whatever the value of $f(\sigma,-\rho \partial \psi / \partial z)$ is. The set of equations Eq. (8) are such that inequality Eq. (7) is systematically satisfied.

According to Eq. (1), and assuming that $\rho \approx \rho_{i}$ whatever the state is, the Cauchy stress tensor $\sigma$ and the evolution equations of the internal, state variables, $\dot{\boldsymbol{\epsilon}}^{p z}$ and $\dot{z}$, explicitly read $(\boldsymbol{G}$ denotes the metric tensor):

$$
\begin{gathered}
\boldsymbol{\sigma}=\lambda \operatorname{Tr}\left(\boldsymbol{\epsilon}-\boldsymbol{\epsilon}^{p z}\right) \boldsymbol{G}+2 \mu\left(\boldsymbol{\epsilon}-\boldsymbol{\epsilon}^{p z}\right)-(3 \lambda+2 \mu)\left(\alpha_{0}(1-z)+\alpha_{1} z\right)\left(T-T_{i}\right) \boldsymbol{G} ; \dot{\boldsymbol{\epsilon}}^{p z}=\frac{3}{2 \sigma_{e q}} \Lambda \mathbf{d e v}(\boldsymbol{\sigma}) ; \dot{z}=\gamma^{p} \Lambda \\
\quad \text { with } \Lambda=\frac{1-z}{\chi^{p}}\left\langle\sigma_{e q}-\left(\exp \left(\frac{T_{i}-T}{T_{i}}\right) \sigma_{i}^{p}+(3 \lambda+2 \mu)\left(\alpha_{0}-\alpha_{1}\right) \gamma^{p}\left(T-T_{i}\right) \operatorname{Tr}\left(\boldsymbol{\epsilon}-\boldsymbol{\epsilon}^{p z}\right)+\beta \gamma^{p} z\right)\right\rangle \quad(9)
\end{gathered}
$$

The local expression of the heat equation is eventually deduced from the expression of the entropy and from Eqs. (1), (2) and (5)-1, namely ( $\Delta$ denotes the Laplacian):

$$
\begin{gathered}
\rho_{i} C_{\epsilon} \frac{T}{T_{i}} \dot{T}-k \Delta T=-(3 \lambda+2 \mu)\left(\alpha_{0}(1-z)+\alpha_{1} z\right) T \operatorname{Tr}(\dot{\boldsymbol{\epsilon}})+ \\
\left(\boldsymbol{\sigma}+(3 \lambda+2 \mu)\left(\alpha_{0}(1-z)+\alpha_{1} z\right) T \boldsymbol{G}\right): \dot{\boldsymbol{\epsilon}}^{p z}-\left((3 \lambda+2 \mu)\left(\alpha_{1}-\alpha_{0}\right) T_{i} \operatorname{Tr}\left(\boldsymbol{\epsilon}-\boldsymbol{\epsilon}^{p z}\right)+\beta z\right) \dot{z}
\end{gathered}
$$

\section{A $1 D$ illustrative example}

As an illustration of the potentialities of the proposed thermo-mechanical model, see Section 2, we consider the problem of a cubic sample (edge: $10 \mathrm{~mm}$ ) of steel subjected to a quasi-static (no inertia effects), cyclic loading. It is assumed that, due to the friction between the sample and the loading apparatus, the temperature varies on the surface of the sample where the loading is applied (and therefore, by heat conduction, in the whole sample). From an experimental point of view, the loading cycle is then defined as follows: $\mathrm{i}$ - simple compression at constant stress rate for $t \in\left[0, t_{1} / 2\right)$, inducing an increase of the temperature due to friction effects; ii - unloading at constant stress rate for $t \in\left[t_{1} / 2, t_{1}\right)$ with a decrease of the temperature due to complex phenomena of heat transfer between the sample and its environment; iii - no mechanical loading for $t \in\left[t_{1}, t_{e q}\right)$, where $t_{e q}$ is such that $T\left(t_{e q}\right)=T_{i}$ in the whole sample (return to thermal equilibrium).

The computation of the temperature variations due to friction and other heat transfer phenomena between the sample and its environment is an involved problem, which is clearly out of the scope of the present 
study: the temperature variations are taken into account in a schematic way, i.e. by a linear approximation between the room temperature $\left(T_{\text {room }}=T_{i}=300 \mathrm{~K}\right)$ and a maximal temperature $\left(T_{L}^{\max }=325 \mathrm{~K}\right)$. Furthermore, the thermo-mechanical model defined in Section 2 reduces to a simple thermo-elastic one (no phase transformation) when $f(\boldsymbol{\sigma},-\rho(\partial \psi / \partial z)) \leq 0$, which is the case in the considered problem whatever $t \in\left[t_{1}, t_{e q}\right)$ may be. Thus, from a numerical point of view, the third part of the experimental, loading cycle (see iii - above) is omitted, i.e. the numerical, loading cycle is defined as follows (see also Fig.1-b): $\mathrm{i}$ - simple compression at constant stress rate and linear increase of the temperature for $t \in\left[0, t_{1} / 2\right)$; ii - unloading at constant stress rate and linear decrease of the temperature for $t \in\left[t_{1} / 2, t_{1}\right)$. The thermal equilibrium, which is experimentally obtained for $t_{e q} \gg t_{1}$, is numerically simulated by setting $T=T_{i}=300 \mathrm{~K}$ in the whole sample at time $t=t_{1}$.

Assuming, in a first approximation, that the four stress-free surfaces of the sample are also adiabatic and that the temperature field as well as the stress field are uniform on the two other surfaces, the problem reduces to a scalar one: from now on, the sample will then be considered as a bar (see Fig. 1-a). The equations that are to be solved are immediatly obtained from Eqs $(9)$ and $(10)$, i.e. $(u(x, t)$ denotes the displacement field; whatever the function $A$ is, $A,{ }_{x}$ denotes its first derivative with respect to $x$ and $A,{ }_{x x}$, its second derivative):

$$
\begin{gathered}
\sigma=(\lambda+2 \mu)\left(u,{ }_{x}-\epsilon^{p z}\right)-(3 \lambda+2 \mu)\left(\alpha_{0}(1-z)+\alpha_{1} z\right)\left(T-T_{i}\right) \quad ; \quad \dot{\epsilon}^{p z}=\Lambda \frac{\sigma}{|\sigma|} \quad ; \quad \dot{z}=\gamma^{p} \Lambda \\
\Lambda=\frac{1-z}{\chi^{p}}\left\langle|\sigma|-\left(\exp \left(\frac{T_{i}-T}{T_{i}}\right) \sigma_{i}^{p}+(3 \lambda+2 \mu)\left(\alpha_{0}-\alpha_{1}\right) \gamma^{p}\left(T-T_{i}\right)\left(u,{ }_{x}-\epsilon^{p z}\right)+\beta \gamma^{p} z\right)\right\rangle \\
\rho_{i} C_{\epsilon} \frac{T}{T_{i}} \dot{T}-k T,{ }_{x x}=-(3 \lambda+2 \mu)\left(\alpha_{0}(1-z)+\alpha_{1} z\right) T \dot{u},{ }_{x}+ \\
\left(\sigma+(3 \lambda+2 \mu)\left(\alpha_{0}(1-z)+\alpha_{1} z\right) T\right) \dot{\epsilon}^{p z}-\left((3 \lambda+2 \mu)\left(\alpha_{1}-\alpha_{0}\right) T_{i}\left(u,{ }_{x}-\epsilon^{p z}\right)+\beta z\right) \dot{z}
\end{gathered}
$$

Neglecting the gravity effects, the equation of quasi-static equilibrium reduces to $\sigma,{ }_{x}=0$ : the stress field $\sigma(x, t)$ is uniform, i.e., whatever $x \in[0, L]$ is, $\sigma(x, t)=\sigma_{L}(t)$. The equations Eq. (11) and that of quasistatic equilibrium are solved numerically using a finite different scheme with an explicit time integration (spatial step $\Delta L$; time step $\Delta t$ ). Except $T(x, 0)=T_{i}$, all the initial fields are zero. The boundary conditions are summarized on Fig. 1-b; the loading cycle is applied fifth times. The values of the materialparameters are the following one: $C_{\epsilon}=450 \mathrm{~J}_{\mathrm{kg}}^{-1} \cdot \mathrm{K}^{-1} ; \lambda=115.10^{3} \mathrm{MPa} ; \mu=77.10^{3} \mathrm{MPa} ; \rho_{i}=$ $78 \cdot 10^{-7} \mathrm{~kg} \cdot \mathrm{mm}^{-3} ; \alpha_{0}=176 \cdot 10^{-5} \mathrm{~K}^{-1} ; \alpha_{1}=120.10^{-5} \mathrm{~K}^{-1} ; k=5 \cdot 10^{-2} \mathrm{~W} \cdot \mathrm{mm}^{-1} \cdot \mathrm{K}^{-1} ; \beta=500 \mathrm{MPa}$; $\sigma_{i}^{p}=76 M P a ; \gamma^{p}=1 ; \chi^{p}=1 M P a$.

Results of the numerical simulations are illustrated on Fig. 2. Note that three different values of the spatial step have been tested, namely $\Delta L=L / 50, \Delta L=L / 100$ and $\Delta L=L / 150$ with $L=10 \mathrm{~mm}$. Then, it appeared that, as soon as the classical stability condition $\Delta t<\left(\Delta x^{2} \rho C_{\epsilon}\right) /(2 K)$ is satisfied, the results are "mesh independent". Thus, the length $l_{z}$ of the part of the bar where $z \neq 0$, i.e. where "new" phase is formed by the transformation, is such that $l_{z} \approx 0.15 \mathrm{~L}=1.5 \mathrm{~mm}$; it is the same whatever the cycle is (see Fig. 2-a). This part is localized on the immediate vicinity of the end of the bar where the thermomechanical loading is applied (the left one in the present case), which is one of the main characteristics of TTS, see Section 2. It can be also observed on Fig. 2-a that the maximum value of $z$, which is obtained in $x=0$, increases with the number of cycles. Note, however, that $z<1$, i.e. that the material is actually a mixture of initial and "new" phases. For a point located inside the part of the bar where $z \neq 0$, namely $x=L / 100=0.1 \mathrm{~mm}$, and for the last loading cycle, the stress-strain curve and the associated stress-plastic strain curve are presented on Fig. 2-b. Due to the previous loading cycles, during which the phase transformation has appeared and developped, the strain at the initial time of the cycle is non zero (point $A$ ). The response of the material is purely thermo-elastic between points $A$ and $B$ (resp. points $C$ and $D$ ), where the nonlinearity of the stress-strain curve is only due to thermal effects. The line segment $[D, E]$ corresponds to the return to thermal equilibrium at zero stress. 

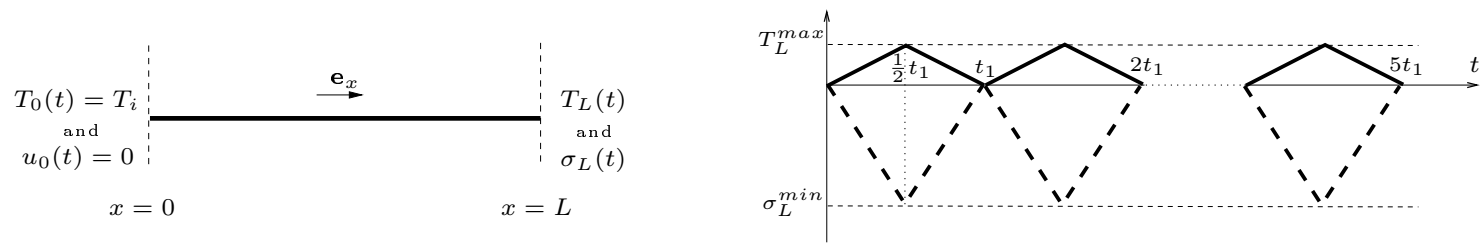

Figure 1: - a (left) Bar subjected to a thermo-mechanical loading: geometry $(L=10 \mathrm{~mm})$ and boundary conditions $\left(T_{i}=300 \mathrm{~K}\right)$. The "loading" functions $T_{L}(t)$ and $\sigma_{L}(t)$ are defined on Figure 1-b. Note that, from a thermal point of view, the lateral surfaces of the bar are considered as adiabatic; - b (right) "Loading" functions $T_{L}(t)$ (solid lines; $T_{L}^{\max }=325 \mathrm{~K}$ ) and $\sigma_{L}(t)$ (dashed lines; $\sigma_{L}^{\min }=-75 \mathrm{MPa}$ ) cyclically applied in $x=0$. The period of the functions is $t_{1}=0.2 \mathrm{~s}$. Note also that $T$ is set equal to $T_{i}$ in the whole bar for $t=n t_{1}$, whatever $n \in\{1,2,3,4,5\}$ is (return to thermal equilibrium).
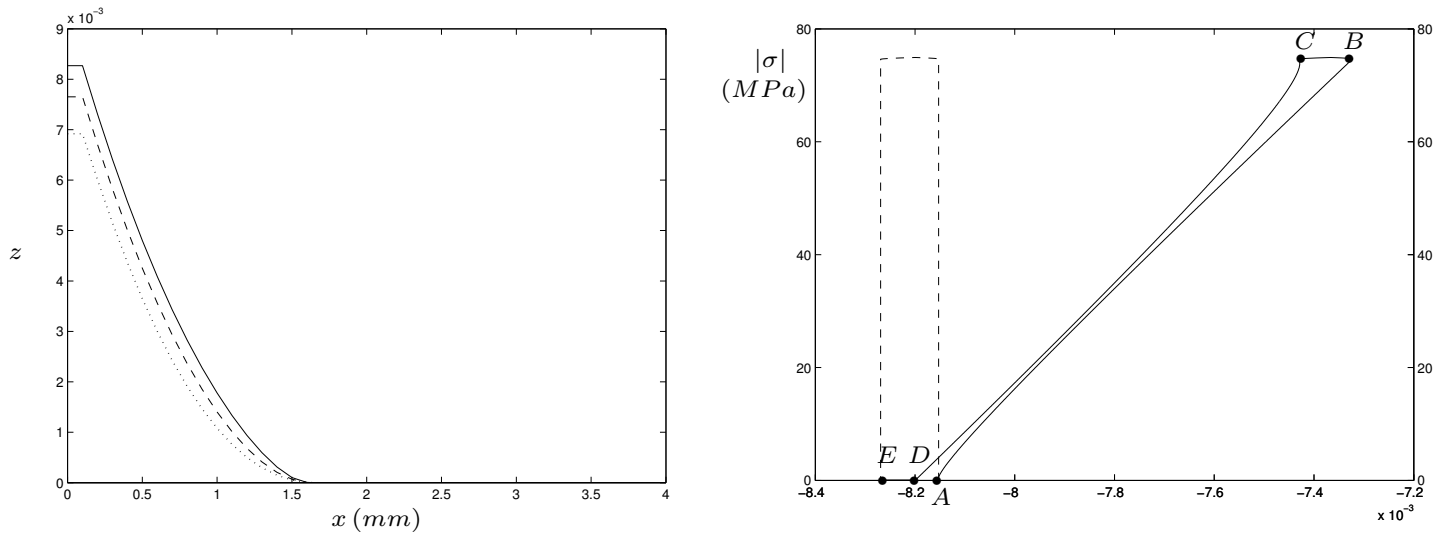

Figure 2: - a (left) Field of the volume fraction of "new" phase in the left part of the bar at the end of the first loading cycle $\left(t=t_{1}=0.2 \mathrm{~s}\right.$; dotted line), of the second cycle ( $t=0.4 \mathrm{~s}$; dashed line) and of the fifth cycle $\left(t=1 \mathrm{~s}\right.$; solid line). Note that the length $l_{z}$ of the part of the bar where the phase transformation occurs is the same whatever the cycle is, namely $l_{z} \approx 0.15 L=1.5 \mathrm{~mm} ;-\mathrm{b}$ (right) Stress-strain curve (solid line) and associated stress-plastic strain curve (dashed line) in $x=L / 100$ and for the fifth loading cycle. Between points $A$ and $B$ (resp. $C$ and $D$ ) of the stressstrain curve, the response of the material is purely thermo-elastic; it is thermo-elasto-viscoplastic with phase transformation between points $B$ and $C$.

\section{Conclusion}

Associated with quasi-static equilibrium and heat equations, the thermo-mechanical model proposed in this study appears to be able to simulate the main characteristics of TTS, i.e. the emergence, on the immediate vicinity of the surface(s) where a thermo-mechanical loading is applied, of a thin layer where the solid-solid phase transformation is localized. More realistic numerical simulations, however, still remain to be done for the relevance of the model to be well established. Experimental works will have also to be performed for the values of some materiel-parameters $\left(e . g . \beta, \gamma^{p}\right.$ and $\chi^{p}$ ) to be precisely identified.

\section{References}

[1] G. Baumann, H.J. Fecht and S. Liebelt, Formation of white-etching layers on rail treads, Wear, 191 (1996), 133-140.

[2] A. Eleöd, F. Oucherif, J. Devecz and Y. Berthier, Conception of numerical and experimental tools for study of the Tribological Transformation of Surface (TTS), in: D. Dowson et al. (Eds), Proc. Lubrication at the Frontier: The Role of the Interface and Surface Layers in the Thin Film and Boundary Regime, Lyon, Elsevier Science, 1999, pp. 673-682.

[3] L. Taleb and F. Sidoroff, A micromechanical modeling of the Greenwood-Johnson mechanism in transformation induced plasticity, Int. J. Plasticity, 19 (2003), 1821-1842.

[4] J. Garrigues, Fondements de la mécanique des milieux continus, Hermes Science Publications, Paris, 2007. 